\title{
Power optimized variation aware dual-threshold SRAM cell design technique
}

This article was published in the following Dove Press journal:

Nanotechnology, Science and Applications

9 February 20II

Number of times this article has been viewed

\author{
Aminul Islam' \\ Mohd Hasan² \\ 'Department of Electronics and \\ Communication Engineering, Birla \\ Institute of Technology, Mesra, Ranchi, \\ Jharkhand, India; ${ }^{2}$ Department \\ of Electronics Engineering, \\ Aligarh Muslim University, Aligarh, \\ Uttar Pradesh, India
}

\begin{abstract}
Bulk complementary metal-oxide semiconductor (CMOS) technology is facing enormous challenges at channel lengths below $45 \mathrm{~nm}$, such as gate tunneling, device mismatch, random dopant fluctuations, and mobility degradation. Although multiple gate transistors and strained silicon devices overcome some of the bulk CMOS problems, it is sensible to look for revolutionary new materials and devices to replace silicon. It is obvious that future technology materials should exhibit higher mobility, better channel electrostatics, scalability, and robustness against process variations. Carbon nanotube-based technology is very promising because it has most of these desired features. There is a need to explore the potential of this emerging technology by designing circuits based on this technology and comparing their performance with that of existing bulk CMOS technology. In this paper, we propose a low-power variation-immune dual-threshold voltage carbon nanotube field effect transistor (CNFET)-based seven-transistor (7T) static random access memory (SRAM) cell. The proposed CNFET-based 7T SRAM cell offers $\sim 1.2 \times$ improvement in standby power, $\sim 1.3 \times$ improvement in read delay, and $\sim 1.1 \times$ improvement in write delay. It offers narrower spread in write access time $(1.4 \times$ at optimum energy point [OEP] and $1.2 \times$ at $1 \mathrm{~V})$. It features $56.3 \%$ improvement in static noise margin and $40 \%$ improvement in read static noise margin. All the simulation measurements are taken at proposed OEP decided by the optimum results obtained after extensive simulation on HSPICE (high-performance simulation program with integrated circuit emphasis) environment.
\end{abstract}

Keywords: carbon nanotube field effect transistor (CNFET), chirality vector, random dopant fluctuation (RDF), SNM

\section{Introduction}

The continuous growth of recent mobile and portable devices and applications has caused a tremendous thrust for low power circuit design. Static random access memory (SRAM) is a highly used circuit in modern processors and occupies a considerable portion of chip area. It is a major contributor to the total power consumption. Various methods and techniques, such as SRAM's cell voltage collapsing, increasing virtual ground, ${ }^{1-3}$ have been applied successfully in the low power and performance region of the design spectrum. However, in some applications where ultralow power consumption is the primary requirement and performance is of secondary importance, a more aggressive approach is warranted. Such applications range from various medical applications (such as implantable pacemakers, defibrillators, hearing aids) to the emerging mobile applications (such as wearable wristwatch computers, wireless sensor nodes, radio-frequency identification [RFID] tags). However, such minimum
Correspondence: Aminul Islam Department of Electronics and Communication Engineering, Birla Institute of Technology

Tel $+9|57| 272$ | $|4|$

Fax +9| 57| 272 || 48

Email aminulislam@bitmesra.ac.in 
energy operation is only possible in the subthreshold region. The pitfall of subthreshold design and near-threshold computing is increased sensitivity to parameter variation. These fluctuations are more pronounced in minimum-geometry devices commonly used in area-constraint circuits such as SRAM cells. ${ }^{4}$ The static noise margin (SNM) model ${ }^{5}$ assumes identical device threshold voltage across all cell transistors, making it unsuitable for predicting the effects of threshold voltage mismatch between adjacent transistors within a cell due to random dopant fluctuation (RDF). Therefore, reinvestigation is required to reassess SNM in scaled technologies to ensure stability of the SRAM cell. Process, voltage, and temperature (PVT) variations can be mitigated by the adaptive body biasing design technique, ${ }^{6}$ but its effect diminishes with technology scaling. ${ }^{7}$ SRAM is a highly used circuit of modern chips. As per the prediction of the International Technology Roadmap For Semiconductors (ITRS), an embedded cache will occupy $90 \%$ of a system on a chip by $2013 .{ }^{8}$ Hence, the design and reinvestigation of the SRAM cell in terms of its design metrics is not only vital, but its robustness against PVT variations is necessary in a nanoscale regime such as $22 \mathrm{~nm}$ technology node.

Research over the past 5 years has demonstrated that the minimum-energy point (MEP) of the energy-delay curve is attractive. The penalty for operating at the MEP is substantial. We can gain $10 \times$ in performance by increasing energy by $20 \%$ above MEP. The most dominant optimization variable around MEP is the supply voltage. ${ }^{9}$ We explored the design space above MEP and below $V_{\mathrm{DD}}$ (supply voltage) to achieve higher performance at the cost of minimal delay. In this paper, we make the following contributions:

1. In view of the aforesaid ultralow power requirement and variation issue, we propose a fully differential dual-diameter carbon nanotube field effect transistor (CNFET)-based seven-transistor (7T) SRAM cell (hereafter called CNFET-7T) and compare its performance at proposed optimum energy point (OEP), defined as low-power dissipation point, with its complementary metal-oxide semiconductor (CMOS) counterpart.

2. We demonstrate that the proposed design outperforms its CMOS counterpart CMOS-based 7T SRAM cell (hereafter called 7T) in terms of static noise margin (SNM) and read-static-noise margin (RSNM).

3. We establish that the proposed design offers improved write access time variation exhibiting its immunity against PVT variation. This is achieved by proper selection of chirality vector for appropriate transistor in the proposed design.
4. In standby mode, SRAM cells are inactive and consume power for data retention due to various leakage components. We investigate leakage power consumed by proposed design and $7 \mathrm{~T}$.

\section{Estimation of threshold voltage of CNFET}

Sixty-five nanometer technology became common after 2006, and $45 \mathrm{~nm}$ technology was announced in 2007. As CMOS continues to scale deeper into the nanoscale, various nonidealities cause the $\mathrm{I}-\mathrm{V}$ characteristics of nanoscaled devices to be substantially different from that of long-channel devices. It becomes more difficult to further improve device/ circuit performance by reducing the physical gate length. As CMOS reaches its scaling limits, development of alternative technologies is necessary to further improve device/ circuit performance by reducing the physical gate length. ${ }^{10}$ Nanotechnology-based fabrication is expected to offer the extra density and potential performance to take electronic circuits to the next step. Several nanoscale electronic devices have been demonstrated in the recent past by researchers, some of the most promising being CNFET. The CNFETs can be scaled down to $10-\mathrm{nm}$ channel length and 4-nm channel width, thereby enhancing throughput in terms of speed and power compared with metal-oxide-semiconductor field-effect transistor (MOSFET). The shortcoming of the CNFET-based design is that there are some fabrication issues, which are likely to be overcome in the near future. The performance of CNFET has significantly improved since the first fabricated device in 1998. Most of the fabrication issues like positioning and alignment of carbon nanotubes (CNTs) along with the presence of metallic CNTs have been solved. ${ }^{11-15}$ The impact of CNT diameter and source/drain doping variations is not significant. ${ }^{16,17}$ Moreover, CNFET can be fabricated using the existing silicon MOSFET infrastructure and it can also be integrated with silicon MOSFET on the same chip. ${ }^{18}$ Most of the fabrication issues have been solved and CNFET technology is promising. It is also important to investigate the potential of systems designed in upcoming technology for their rapid commercialization once the technology matures.

Most of the fundamental limitations of traditional silicon MOSFETs are mitigated in CNFET. With ultralong $(\sim 1 \mu \mathrm{m})$ mean free path for elastic scattering, a ballistic or near-ballistic transport can be obtained with the use of CNTs under low voltage bias to achieve the ultimate device performance. ${ }^{19-21}$ Its quasi-1D structure provides better electrostatic control over the channel region than $3 \mathrm{D}$ devices 
(for example, bulk CMOS) and 2D device (for example, fully depleted SOI (silicon on insulator)) structures. ${ }^{22}$ Ballistic transport operation and low $\mathrm{I}_{\mathrm{OFF}}$ (OFF-current) make the CNFET a suitable device for high performance and increased integration. CNTs are categorized into single-walled CNTs (SWCNTs) and multiwalled CNTs (MWCNTs). Most SWCNTs have a diameter of close to $1 \mathrm{~nm}$, with a tube length that can be many millions of times longer. The structure of a SWCNT can be conceptualized by wrapping a 1-atom-thick layer of graphite called graphene into a flawless cylinder. SWCNT-based CNFETs are used in this design.

Properties of a SWCNT depend on its chirality $\left(n_{1}, n_{2}\right)$ : the direction in which it is rolled up. The CNT acts as metal if $n_{1}=n_{2}$ or $\left(n_{1}-n_{2}\right) / 3=i$, where $i$ is an integer. Otherwise, the CNT works as semiconductor. The $D_{\mathrm{CNT}}$ (diameter of CNT) is estimated using its chirality vector $\left(n_{1}, n_{2}\right)$ as: ${ }^{23-26}$

$$
D_{C N T}=\frac{a}{\pi} \sqrt{n_{1}^{2}+n_{2}^{2}+n_{1} n_{2}}
$$

The $V_{\mathrm{t}}$ (threshold voltage) of CNFET can be approximated to the first order as the half bandgap, which is an inverse function of the CNT diameter $\left(D_{\mathrm{CNT}}\right):{ }^{23}$

$$
V_{t} \approx \frac{E_{g}}{2 q}=\frac{1}{\sqrt{3}} \frac{a V_{\pi}}{q D_{C N T}}
$$

where $E_{\mathrm{g}}=$ energy gap, $q=$ electronic charge, $a=\sqrt{ } 3 d=$ $2.49 \AA$ is the lattice constant (where $d \approx 1.44 \AA$ is the inter-carbon-atom distance) and $V_{\pi}=3.033 \mathrm{eV}$ is the carbon $\pi$-to- $\pi$ bond energy in the tight bonding model. The use of appropriate $D_{\mathrm{CNT}}$ and hence $V_{\mathrm{t}}$ of CNFETs is a critical piece of our design strategy. In this work, dual- $V_{\mathrm{t}}$ and dual-diameter CNFETs are used using chiral vector values $(11,0)$ and $(13,0)$. The $D_{\mathrm{CNT}}$ of the CNFET with chiral vector value $(11,0)$ and $(13,0)$ are computed using $(1)$ to be $0.8719 \mathrm{~nm}$ (MP1, MP2, MN1, MN2 and MN4 of CNFET-7T) and $1.03 \mathrm{~nm}$ (for MN3 and MN5 of CNFET-7T) respectively. The $V_{\mathrm{t}}$ of the CNFET with chiral vector value of $(11,0)$ and $(13,0)$ are computed using (2) to be $0.5018 \mathrm{~V}$ and $0.4246 \mathrm{~V}$ respectively.

Compared with CMOS circuits, the CNFET circuits with 1-10 CNTs per device is about 2 to 10 times faster. ${ }^{10,25}$ CNTs are placed on substrate having a dielectric constant of $\mathrm{K}_{\text {sub }}=4$. The tubes are separated by a high-k (Hi-k) material called $\mathrm{HfO}_{2}$ (hafnium oxide) having a dielectric constant of $\mathrm{K}_{\mathrm{ox}}=16$ and thickness of $\mathrm{t}_{\mathrm{ox}}=4 \mathrm{~nm}$. Other important device and technology parameters related to CNFET are tabulated in Table 1. The I-V characteristics of employed CNFETs with chirality vector $(11,0)$ and $(13,0)$ and NMOS (n-type metal-oxide-semiconductor) with zero bias threshold
Table I Device and technology parameters of CNFET

\begin{tabular}{lll}
\hline Parameter & Description & Value \\
\hline $\mathrm{L}_{\mathrm{ch}}$ & Physical channel length & $22 \mathrm{~nm}$ \\
$\mathrm{~W}_{\mathrm{g}}$ & The width of metal gate (sub_pitch) & $6.4 \mathrm{~nm}$ \\
$\mathrm{~K}_{\mathrm{ox}}$ & Dielectric constant of high-K gate oxide & 16 \\
$\left(\mathrm{n}_{1}, \mathrm{n}_{2}\right)$ & Chirality of the tube & $(\mathrm{II}, 0)$ \\
& & $(\mathrm{I}, 0)$ \\
$\mathrm{n} \_\mathrm{CNT}$ & Number of tube & $\mathrm{I}$
\end{tabular}

voltage, $V_{\mathrm{tn} 0}=0.63 \mathrm{~V}$ are plotted in Figure 1. P-type CNFET used in the proposed design has $\mathrm{I}-\mathrm{V}$ characteristics with opposite polarity (not shown). The threshold voltages of N-CNFET are estimated using (1) and (2) with chirality vector ranging from $(7,0)$ to $(36,0)$. First, the $D_{\mathrm{CNT}}$ are estimated substituting the value of the constant $\pi=3.142$, the value of the lattice constant $a=2.49 \AA$, and the value of $n_{1}$ ranging from 7 to 36 keeping $n_{2}=0$. Next, the threshold voltage $V_{\mathrm{t}}$ is estimated substituting the value of $a=2.49 \AA$, the value of the carbon $\pi-\pi$ bond energy $V_{\pi}=3.033 \mathrm{eV}$, the value of electronic charge $q=1.6 \times 10^{-19} \mathrm{C}$, and the estimated value of $D_{\mathrm{CNT}}$. The estimated values of threshold voltage for each value of $n_{1}$ ranging from 7 to 36 are plotted in Figure 2 . The plot in Figure 2 shows two end points with $V_{\mathrm{t}}=0.78857 \mathrm{~V}$ at $n_{1}=7$ and $V_{\mathrm{t}}=0.153 \mathrm{~V}$ at $n_{1}=36$. Two other important points in this plot are $(11,0.5018)$ and $(13,0.4246)$ which indicate $V_{\mathrm{t}}=0.5018 \mathrm{~V}$ at $n_{1}=11$ and $V_{\mathrm{t}}=0.4246 \mathrm{~V}$ at $n_{1}=13$. These are the threshold voltages of CNFETs used in the proposed design. The threshold voltage $\left(V_{\mathrm{t}}\right)$ of P-type CNFET employed in this design has an opposite polarity. ${ }^{27}$

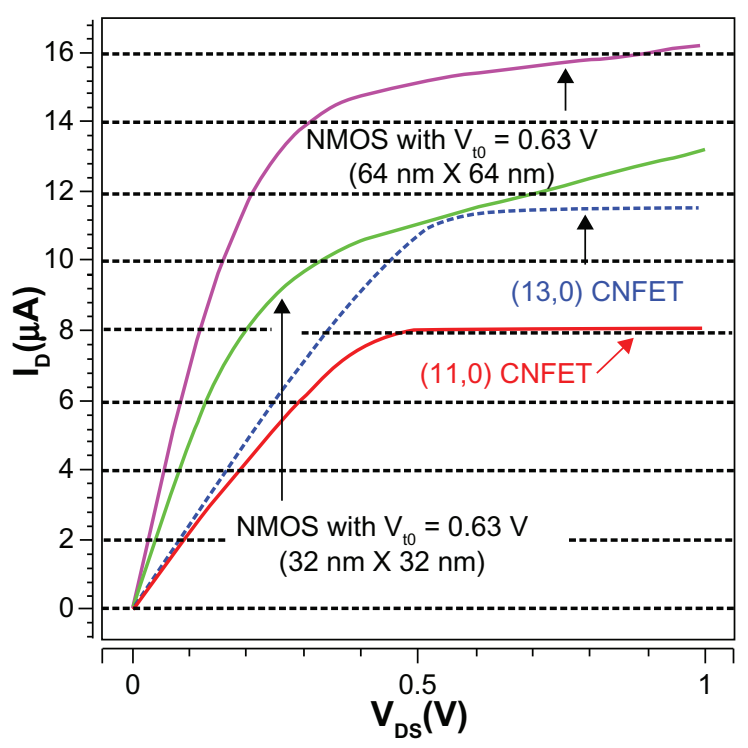

Figure I Comparison of I-V characteristics of NMOS and CNFET.

Abbreviations: CNFET, carbon nanotube field effect transistor; NMOS, n-type metal-oxide-semiconductor. 


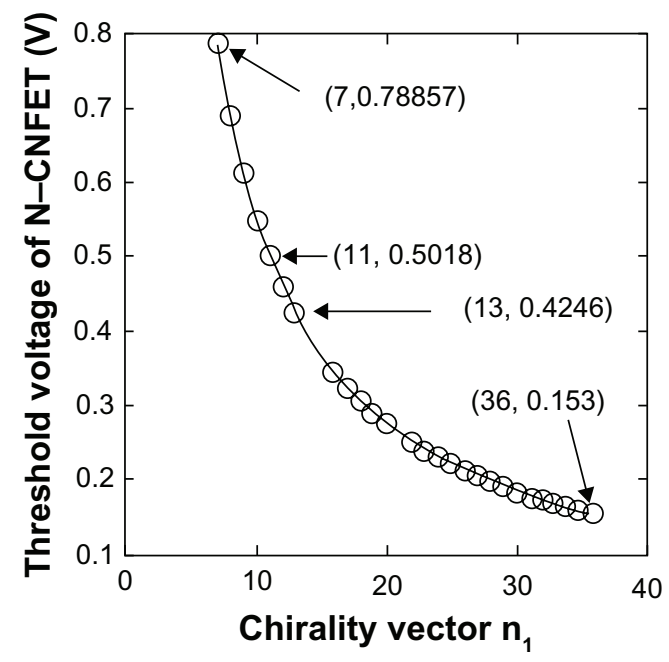

Figure 2 Threshold voltage $\left(V_{t}\right)$ versus chirality vector $\left(n_{1}\right)$. Abbreviation: CNFET, carbon nanotube field effect transistor.

\section{Proposed CNFET-based 7T SRAM cell}

Aly et $\mathrm{al}^{28}$ proposed a CMOS-7T SRAM cell similar to the proposed CNFET-7T shown in Figure 3 (or Figure 4), basically to reduce the activity factor $\alpha_{B L}$ for reduction of dynamic power while writing to a cell given by $P_{\text {WRITE }}=\alpha_{\mathrm{BL}} \times C_{\mathrm{BL}} \times V_{\mathrm{DD}}^{2} \times F_{\text {WRITE }}$, where $\alpha_{\mathrm{BL}}$ is the switching activity factor for writing, $C_{\mathrm{BL}}$ is the bitline capacitance, $V_{\mathrm{DD}}$ is the supply voltage, and $F_{\text {WRITE }}$ is the writing frequency to the SRAM cell. But static power consumption in a SRAM cell is more critical than dynamic power consumption, since whole parts of the cache remains idle most of the time except the row being accessed. Therefore, this paper proposes implementation of 7T SRAM with CNFET operating at OEP and demonstrates that the proposed design is better in terms of standby power dissipation.

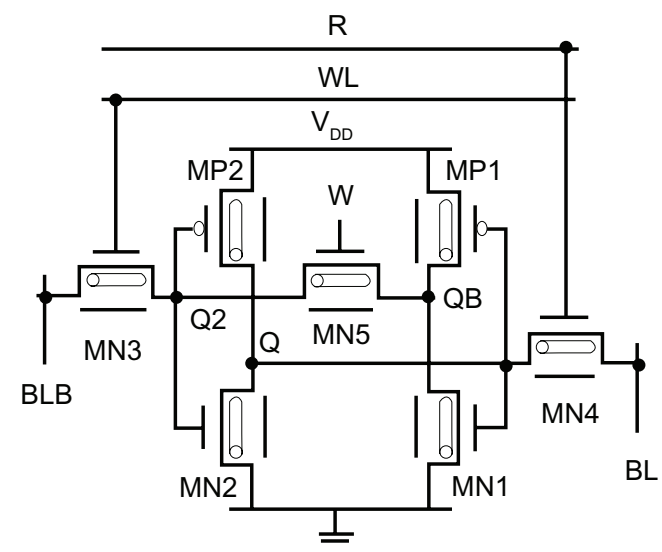

Figure 3 CNFET-based 7T SRAM cell.

Abbreviation: CNFET, carbon nanotube field effect transistor; SRAM, static random access memory.

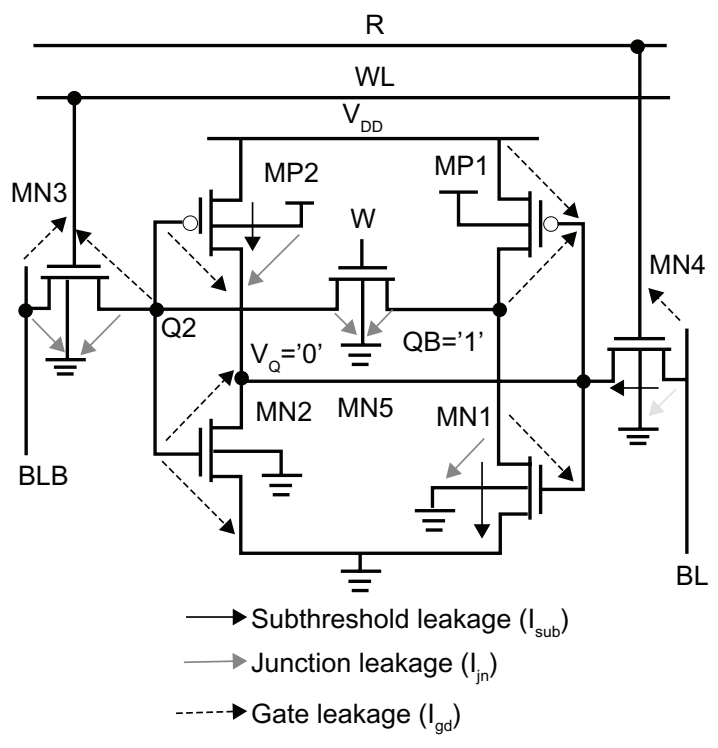

Figure 4 Leakage components in 7T SRAM cell.

Abbreviations: 7T, seven-transistor; SRAM, static random access memory.

\section{Read/write operation}

Both BL and BLB are precharged high before and after each $\mathrm{read} / \mathrm{write}$ operation. The write operation in the 7T/CNFET-7T cell starts by turning MN5 off. Complement of data to be written to node Q is applied to BLB and MN3 is turned on by asserting WL high, leaving MN4 off. BL and MN4 do not take part in the write operation. During standby mode, MN3 and MN4 are kept off applying WL and R low, MN5 is kept on asserting W high. During read operation with QB storing “0”, BLB discharges through the critical read path consists of MN3, MN5, and MN1. BL discharges through the read path MN4 and MN2 during read operation with Q storing “" 0 ”. Main device and technology parameters for 7T cell used for comparison with the proposed design are tabulated in Table 2 . As MN1 $\geq 3$ and MN2 $\geq 2$ ensure stable read operation, ${ }^{28}$ the transistors in the $7 \mathrm{~T}$ cell are sized as shown in Table 2. The 7T also requires low- $V_{\mathrm{t}} \mathrm{MN5}$. To avoid extra masking cost and to fulfill this requirement, the diameter of MN5 is increased to reduce its $V_{\mathrm{t}}$ in the proposed design. Other devices used for the design are square transistors with

Table 2 Device and technology parameters of CMOS-based 7T

\begin{tabular}{ll}
\hline Parameter & CMOS-based 7T \\
\hline$V_{\mathrm{tn} 0}$ & $0.63 \mathrm{~V}$ \\
$V_{\mathrm{tp0}}$ & $-0.5808 \mathrm{~V}$ \\
$\mathrm{MPI}, \mathrm{MP2}$ & $\mathrm{W}=22 \mathrm{~nm}, \mathrm{~L}=22 \mathrm{~nm}$ \\
MNI & $\mathrm{W}=66 \mathrm{~nm}, \mathrm{~L}=22 \mathrm{~nm}$ \\
MN2 & $\mathrm{W}=44 \mathrm{~nm}, \mathrm{~L}=22 \mathrm{~nm}$ \\
MN3, MN4, MN5 & $\mathrm{W}=22 \mathrm{~nm}, \mathrm{~L}=22 \mathrm{~nm}$ \\
\hline
\end{tabular}

Abbreviations: CMOS, complementary metal-oxide semiconductor; 7T, seventransistor; W, width; L, length. 
minimum channel length. This work implements CMOSbased 7T SRAM cell using dual-diameter dual- $V_{t}$ CNFETs, and its performance is assessed at OEP.

To investigate the performance in terms of various design metrics, extensive simulations are run on HSPICE (highperformance simulation program with integrated circuit emphasis), and finally CNFETs with two different diameters are selected to achieve optimum results. HSPICE has been an industry-standard circuit simulator for the past 30 years with an error of less than 5\%. As mentioned earlier, the diameter selected for MN3 and MN5 is $1.03 \mathrm{~nm}$ to increase its drive current, as drive current through CNT is proportional to its diameter. This has resulted in reduction of read and write delays, which are shown later. The diameter of the other five transistors is selected to be $0.8719 \mathrm{~nm}$. The values of device diameters, threshold voltages, and the corresponding chirality vectors are tabulated in Table 3. As mentioned earlier, single-tube CNFETs are used in this work.

\section{Simulation results and comparisons}

We present measurements of various design metrics which are measured during simulation on HSPICE using experimentally validated CNFET model, ${ }^{23}$ and $22 \mathrm{~nm}$ CMOS Berkeley Predictive Technology Model (BPTM) ${ }^{29}$ The CNFET model has been calibrated to $90 \%$ accuracy with experimental data (AC and DC characteristics) from fabricated CNFET circuits. $^{30}$

\section{Standby power}

The leakage current is the major contributor to the total power consumption in SRAM cell in nanoscaled technology. The total leakage current in a SRAM cell is mainly (neglecting minor components such as $I_{\mathrm{GIDL}}$ and $\left.I_{\text {punchthrough }}\right)$ due to subthreshold-leakage current $\left(I_{\text {sub }}\right)$, the gate-leakage current $\left(I_{\text {gate }}\right)$ and the reverse-biased drain- and source-body junction band-to-band tunneling (BTBT) leakage current $\left(I_{\text {jn }}\right)$ through different transistors as shown in Figure $4:^{31-33}$

$$
\begin{aligned}
& I_{s u b}=I_{s u b M N 4}+I_{s u b M N 1}+I_{s u b M P 2} \\
& I_{j n}=2 I_{j n M N 3}+I_{j n M N 4}+I_{j n M N 1}+I_{j n M P 2}+2 I_{J N M N 5} \\
& I_{\text {gate }}=I_{g d M N 3}+I_{g s M N 3}+I_{g d M N 4}+I_{g d M P 2} \\
& +I_{g d M N 2}+I_{g s M N 2}+I_{g d M P 1}+I_{g s M P 1}+I_{g d M N 1} \\
& I_{\text {leak }}=I_{\text {sub }}+I_{j n}+I_{\text {gate }}
\end{aligned}
$$

Figure 4 shows the various leakage components in 7T, with storage node Q and QB storing the values as indicated. The $S_{\text {PWR }}$ (standby power) consumed due to these leakage
Table 3 Device parameters of proposed design

\begin{tabular}{llll}
\hline CNFETs & $\begin{array}{l}\text { Chirality } \\
\text { vector }\end{array}$ & $\begin{array}{l}\text { Threshold } \\
\text { voltage }(\mathbf{V})\end{array}$ & $\begin{array}{l}\text { Diameter } \\
(\mathbf{n m})\end{array}$ \\
\hline MPI, MP2, MNI, & $(\mathrm{II}, 0)$ & $|0.5018|$ & 0.8719 \\
MN2, MN4 & $(13,0)$ & 0.4246 & 1.03 \\
\hline MN3, MN5 & $(13,0)$
\end{tabular}

Abbreviation: CNFET, carbon nanotube field effect transistor.

currents is measured at various voltage points starting from $V_{\mathrm{DD}}=1 \mathrm{~V}$ to the minimal voltage of $V_{\mathrm{DD}}=857 \mathrm{mV}$ and $660 \mathrm{mV}$ for 7T and CNFET-7T respectively. As the cell is asymmetrical, the measurement is done under two conditions with storage node Q storing " 0 " and Q storing " 1 " because it is evident from Figure 4, that the leakage components, and thus the current equation differ depending on stored value at $\mathrm{Q}$ and $\mathrm{QB}$.

The measured results are averaged and the mean values are plotted in Figure 5, where important measurement points are marked with arrows. Compared with 7T (operating at $V_{\mathrm{DD}}=1 \mathrm{~V}$ consuming $61.94 \mathrm{pW}$ ), the CNFET-7T (operating at $1 \mathrm{~V}$ consuming $57.73 \mathrm{pW}$ ) saves $1.073 \times$ standby power. As observed from Figure 5, the $7 \mathrm{~T}$ consumes $38.54 \mathrm{pW}$ whereas CNFET-7T consumes $33.63 \mathrm{pW}$ at iso-operating voltage of $857 \mathrm{mV}$. Therefore even if the proposed design is operated at the minimum operating voltage of $7 \mathrm{~T}$ (ie, $V_{\mathrm{DD}}=857 \mathrm{mV}$ ) there is a saving in $S_{\mathrm{PWR}}$ by $1.146 \times$. This is attributed to the higher leakage current components in MOSFET compared with CNFET. That is, OFF-current of CNFET is much lower than that of MOSFET. As illustrated in Figure 5, if the CNFET-7T is operated at $660 \mathrm{mV}$, there will be a substantial amount of saving in $S_{\mathrm{PWR}}$.

Under the measuring condition used (with equal wordline slew rate and on time), 7T could be operated up to $857 \mathrm{mV}$ and CNFET-7T could be operated up to $660 \mathrm{mV}$ without

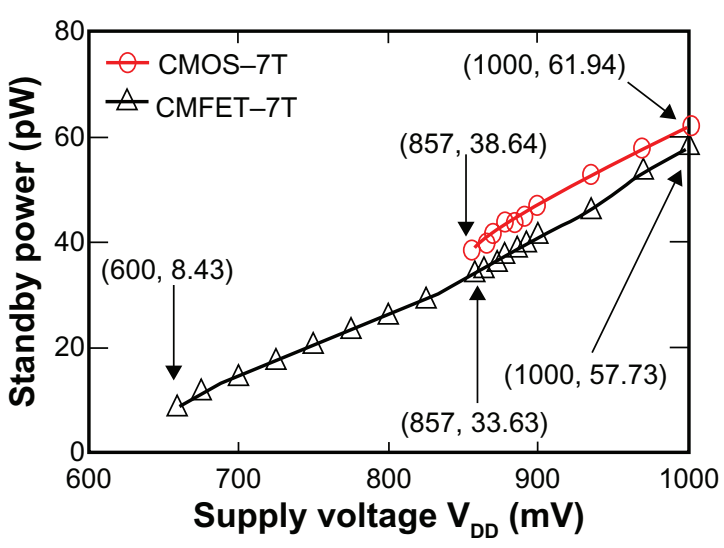

Figure 5 Standby power $\left(S_{P W R}\right)$ measured with Q storing "0".

Abbreviations: CMOS, complementary metal-oxide semiconductor; CNFET, carbon nanotube field effect transistor. 
facing read and write failure. If the measuring condition is changed, both the design can be operated even in the near subthreshold region. Particularly, CNFET-7T can be operated in subthreshold region without failure. However, the designs are operated in the superthreshold region to explore OEP in this region without compromising much with delay penalty. The iso-operating voltage, ie, $857 \mathrm{mV}$, is found to be OEP, without incurring much delay in accessing the cell. $V_{\mathrm{DD}}$ can be further scaled down for CNFET-7T for improved results.

\section{Read/write access time}

Figure 6 shows the read access time $\left(T_{\mathrm{RA}}\right)$ versus $V_{\mathrm{DD}}$ plot. The $T_{\mathrm{RA}}$ is estimated as the time required for developing a voltage differential to be sensed by the sense amplifier (not shown) from the point when WL reaches $50 \%$ of its full swing from its initial low level to the point when BL/BLB falls by $10 \%$ of its full swing from its initial high value. As the cell is asymmetrical, the measurement is done under two conditions - with storage node Q storing " 0 " and Q storing " 1 " and the results are averaged before plotting. It is observed from Figure 6 that the read delay of 7T at iso-operating voltage of $857 \mathrm{mV}$ is $1.321 \times$ compared with CNFET-7T.

Figure 7 shows the write access time $\left(T_{\mathrm{WA}}\right)$ versus $V_{\mathrm{DD}}$ plot. While writing " 0 " to Q, BLB is raised high and the write operation is completed after two inverter delays when QB settles to its new state. Therefore, this operation takes quite a long time compared with the read operation. MN3 of CNFET-7T is of low- $V_{\mathrm{t}}$. Therefore, it passes a higher voltage to the gate of MN2 driving it harder (compared with 7T) both in cases of reading as well as writing, thereby reducing the $\mathrm{read} /$ write delay. The CNFET-7T offers $1.066 \times$ improvement in write delay at iso-operating voltage of $V_{\mathrm{DD}}=857 \mathrm{mV}$. As observed from Figures 5 and 6, operation of CNFET-7T

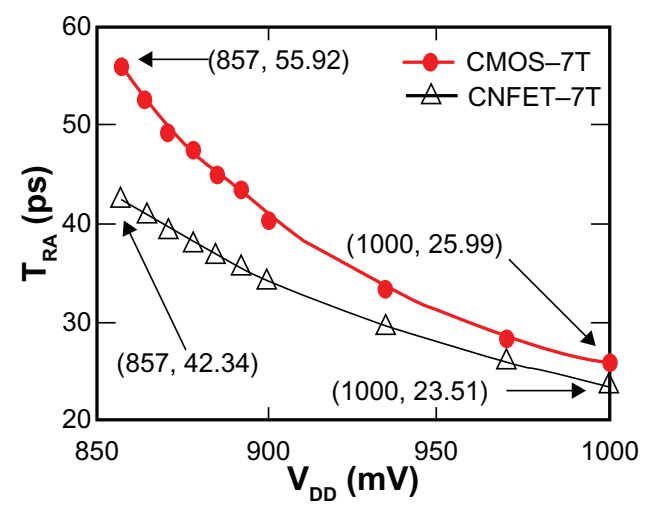

Figure 6 Read access time versus $V_{D D}$.

Abbreviations: CMOS, complementary metal-oxide semiconductor; CNFET, carbon nanotube field effect transistor.

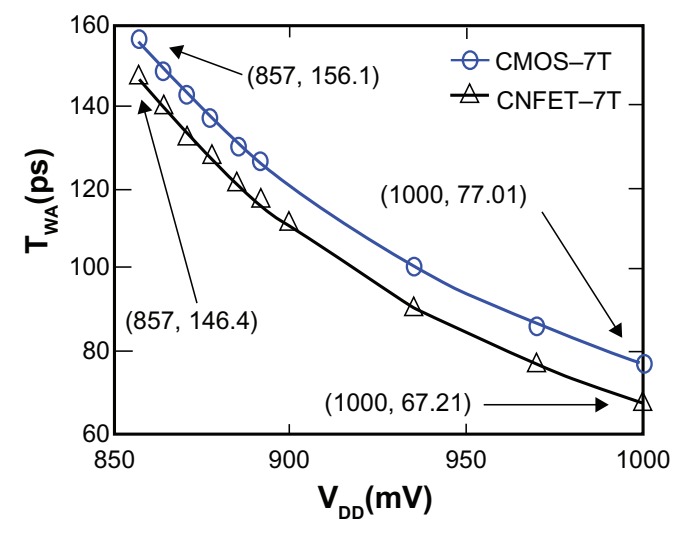

Figure 7 Write access time versus $V_{D D}$.

Abbreviations: CMOS, complementary metal-oxide semiconductor; CNFET, carbon nanotube field effect transistor.

at OEP instead of $V_{\mathrm{DD}}=1 \mathrm{~V}$ offers $41.7 \%$ improvement in $S_{\text {PWR }}$ incurring $44.5 \%$ penalty in $T_{\mathrm{RA}}$, whereas operation of $7 \mathrm{~T}$ at OEP instead of $V_{\mathrm{DD}}=1 \mathrm{~V}$ offers $37.8 \%$ improvement in $S_{\text {PWR }}$ incurring $53.5 \%$ penalty in $T_{\mathrm{RA}}$. Therefore, the proposed design is superior to its CMOS counterpart in terms of most of its major design metrics discussed so far.

\section{Write access time variation}

We present variation measurements of write-access time $\left(T_{\mathrm{wA}}\right)$, which is measured during simulation on HSPICE. Monte Carlo simulations are performed for the measurements. Monte Carlo simulation is a method for iteratively evaluating a design. The goal is to determine how random variation on process parameters, voltage, and temperature affects the performance and reliability of a design. The standard deviation $(\sigma)$ is a measure of dispersion (or variability) that states numerically the extent to which individual observations vary on average. Figure 8 plots the normalized standard deviation of $T_{\mathrm{WA}}$ of the two designs at $\operatorname{OEP}\left(V_{\mathrm{DD}}=0.857 \mathrm{~V}\right)$ and $V_{\mathrm{DD}}=1 \mathrm{~V}$. It is observed from the figure that the proposed design shows $1.4 \times$ less variation in write access time at $0.857 \mathrm{~V}$ and $1.2 \times$ at $1 \mathrm{~V}$. This shows the robustness of the

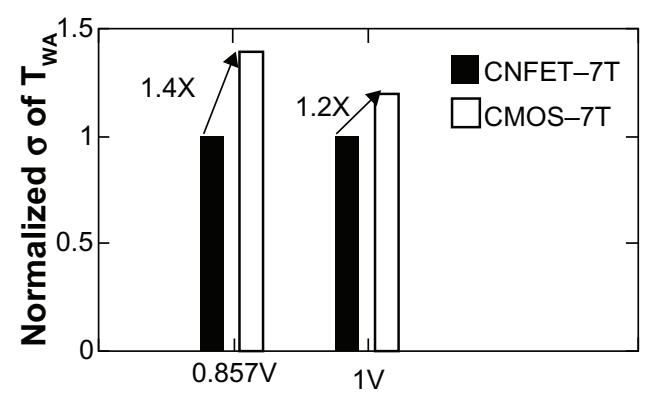

Figure 8 Write delay variation measurement.

Abbreviations: CMOS, complementary metal-oxide semiconductor; CNFET, carbon nanotube field effect transistor. 
proposed design against PVT variations. Ideally $I_{\mathrm{D}}$ is supposed to be constant in saturation region. However, in practice, increasing $V_{\text {DS }}$ (drain-to-source voltage) beyond $V_{\text {DS-sat }}$ (drain-to-source saturation voltage) does affect the channel somewhat. As the $V_{\mathrm{DS}}$ is increased, the depletion layer widens, thereby reducing the effective channel length. Since $I_{\mathrm{D}}$ is inversely proportional to the channel length, $I_{\mathrm{D}}$ increases with $V_{\mathrm{DS}}$. This DIBL (drain-induced barrier lowering) or SCE (short-channel effect) is more pronounced in small geometry MOSFET, and it has negligible impact on CNFET, which is evident from Figure 1. It clearly shows that the $I_{\mathrm{D}}$ in saturation region is almost constant in case of CNFET, whereas it shows almost linear increase in case of MOSFET. This signifies the variation in $I_{\mathrm{D}}$ with $V_{\mathrm{DS}}$. It is needless to say that during $\mathrm{read} /$ write operations node voltages change thereby varying $V_{\mathrm{DS}}$ and finally causing variation in $T_{\mathrm{WA}}$ and $T_{\mathrm{RA}}$.

\section{Static noise margin measurements}

The static noise margin (SNM) of SRAM cell is defined as the minimum DC noise voltage necessary to flip the state of the cell. SNM of an SRAM is a widely used design metric that measures the cell stability. Simulation measurement of SNM is carried out for both the designs. Figure 9 shows a test setup for measuring SNM. Figure 10 plots the combined "butterfly curve" of 7T and proposed CNFET-7T at OEP (iso-operating voltage of $V_{\mathrm{DD}}=857 \mathrm{mV}$ ). The butterfly curve is obtained in the following way using the test circuit: 1) $\mathrm{W}$ and bit lines (BL, BLB) are biased at $V_{\mathrm{DD}}$ and $\mathrm{WL}$ and $\mathrm{R}$ is biased at ground; 2) Voltage of $\mathrm{N} 1$ is swept from $0 \mathrm{~V}$ to $V_{\mathrm{DD}}$ while measuring voltage of QB; 3) Voltage of $\mathrm{N} 2$ is swept from $0 \mathrm{~V}$ to $V_{\mathrm{DD}}$ while measuring voltage of $\mathrm{Q}$ in the same way; and 4) Measured voltages are plotted to get a butterfly curve. The side length of maximum square that can be embedded within the smaller lobe of the butterfly curve represents the SNM of the cell.

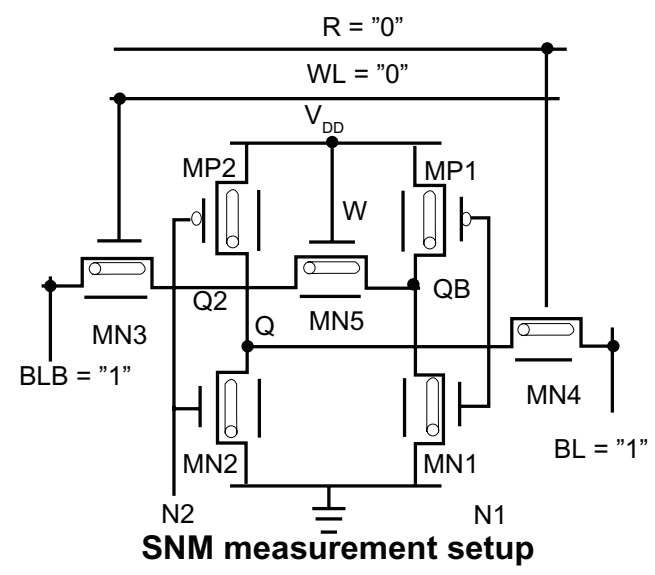

Figure 9 Test setup for measuring static noise margin (SNM).

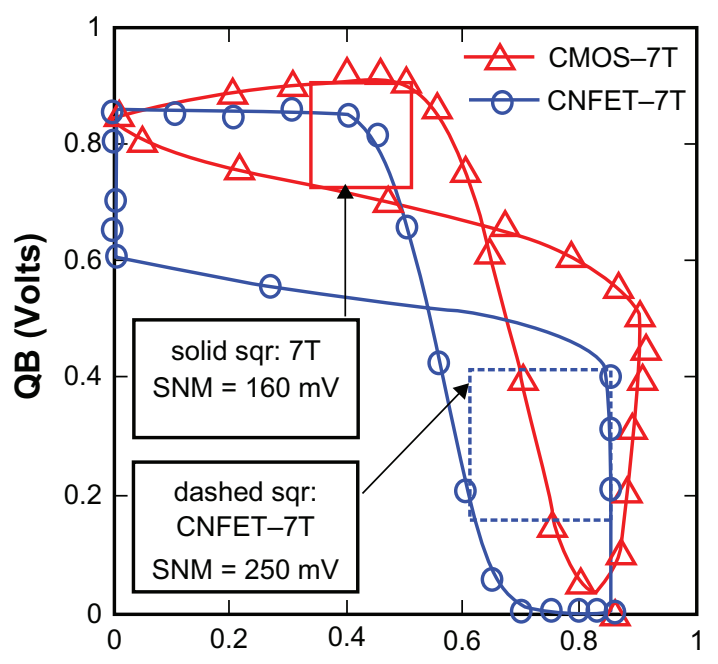

Figure 10 SNM of $7 \mathrm{~T}$ and CNFET-7T.

Abbreviations: CMOS, complementary metal-oxide semiconductor; CNFET, carbon nanotube field effect transistor; SNM, static noise margin.

This definition holds good because, when the value of noise voltage increases from 0 , the VTC (voltage transfer characteristic) for inverter 1 formed with MP1 and MN1 moves to the right and the $\mathrm{VTC}^{-1}$ (inverse VTC) for inverter 2 formed with MP2 and MN2 moves downward. Once they both move by the SNM value, the curves meet at only two points and any further noise flips the cell. ${ }^{34}$ As observed from Figure 10, the SNM of 7T is $160 \mathrm{mV}$, whereas that of CNFET-7T is $250 \mathrm{mV}$, showing $56.3 \%$ improvements in CNFET-7T over the 7T cell. To understand why this happens, remember that both the transistors MN1 and MN2 of CNFET-7T have lower $V_{\mathrm{t}}$, which implies that the switching threshold of both the inverters are lower than that of 7T. This shifts the VTC of inverter 1 to the left and pushes the $\mathrm{VTC}^{-1}$ of inverter 2 down making both the lobes of the butterfly curve wider.

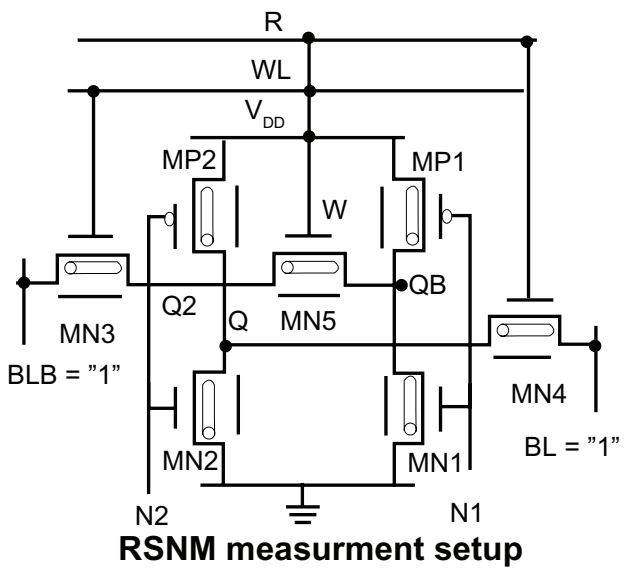

Figure I I Test setup for measuring read static noise margin (RSNM). 


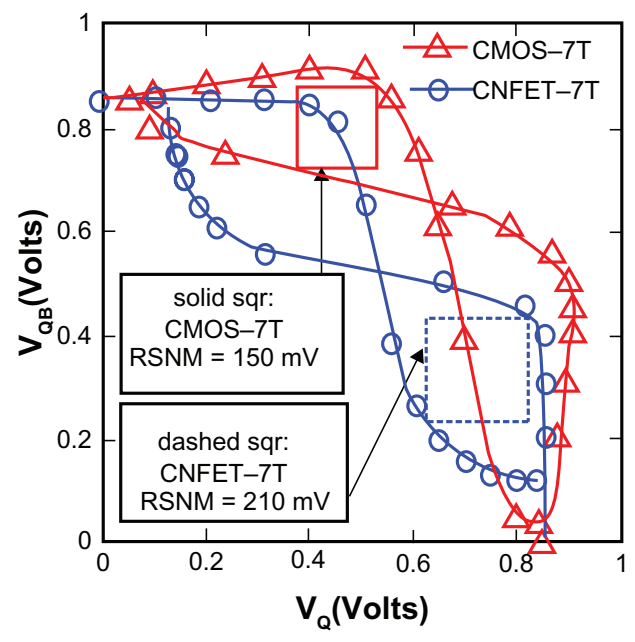

Figure 12 Read static noise margin (RSNM) of 7T and CNFET-7T. Abbreviations: CMOS, complementary metal-oxide semiconductor; CNFET, carbon nanotube field effect transistor.

\section{Read static noise margin measurements}

The SRAM cell is the most susceptible to noise during read operation since the node storing " 0 " (say, Q in Figure 4) rises to a voltage higher than ground due to a voltage dividing effect between the access transistor (say, MN4) and inverter pull-down NMOS driver (say, MN2). The ratio of the widths of the pull-down transistor to the access transistor, commonly referred to as the cell ratio (CR) or $\beta$ ratio, decides how high the " 0 " storage node rises during a read operation. Smaller CRs translate into a larger voltage drop across the pull-down transistor, requiring a smaller noise voltage at the node storing " 0 " to trip the cell. RSNM is a measure of how much noise voltage is required at the node storing " 0 " to flip the state of an SRAM cell while reading. Therefore, RSNM is an even more critical design metric of the SRAM cell than SNM. Figure 11 shows a test setup for measuring RSNM. The butterfly curves are obtained with this test setup and using the same measuring technique as done for SNM. Figure 12 plots the combined butterfly curve of 7T and CNFET-7T at OEP. The plot shows that the CNFET-7T outperforms 7T by $40 \%$ improvement in RSNM. The reason is obvious from the discussion in the preceding subsection.

\section{Conclusion}

We propose a dual-Vt CNFET-based low-power PVT variation immune 7T SRAM cell. We observe during the investigation that the CNFET-based 7T SRAM cell offers better performance in terms of most the design parameters such as standby power and noise margins. The proposed design also shows more robustness against process variations compared with the CMOS-based 7T SRAM cell. This is due to proper selection of CNFET diameter and hence threshold voltage for appropriate transistors. This is also due to the cylindrical geometry of CNFET. A variation in the gate oxide thickness that strongly affects the drive current and capacitance of CMOS transistors has a negligible impact on the CNFET's operation. We have successfully demonstrated that the proposed design will be effective to reduce standby power, $\mathrm{read} / \mathrm{write}$ delay, and write delay variation.

\section{Disclosure}

The authors report no conflicts of interest in this work.

\section{References}

1. Zhang K, Bhattacharya U, Chen Z, et al. A 3-GHz 70-Mb SRAM in 65-nm CMOS technology with integrated column-based dynamic power supply. IEEE J Solid-State Circuits. 2006;41(1):146-151.

2. Nii K, Yabuuchi M, Tsukamoto Y, et al. A 45-nm bulk CMOS embedded SRAM with improved immunity against process and temperature variations. IEEE J Solid-State Circuits. 2008;43(1):180-191.

3. Ohbayashi S, Yabuuchi M, Nii K, et al. A 65-nm SoC embedded 6T-SRAM designed for manufacturability with read and write operation stabilizing circuits. IEEE J Solid-State Circuits. 2007; 42(4):820-829.

4. Burnett D, Erington K, Subramanian C, Baker K. Implications of fundamental threshold voltage variations for high-density SRAM and logic circuits. Proceedings of the Symposium onVLSI Technology; Honolulu, HI: IEEE; 1994 Jun 7-9:15-16.

5. Seevinck E, List F, Lohstroh J. Static-noise margin analysis of MOS SRAM cells. IEEE J Solid-State Circuits. 1987;SC-22:748-754.

6. Tschanz JW, et al. Adaptive body bias for reducing impacts of die-to-die and within-die parameter variations on microprocessor frequency and leakage. IEEE J Solid-State Circuits. 2002;37(11): 1396-1402.

7. Borkar S. Design challenges of technology scaling. IEEE Micro. 1999;19(4):23-29.

8. Semiconductor Industry Association (SIA), International Technology Roadmap for Semiconductors 2005 Edition. Available from: http:// www.itrs.net/Links/2005ITRS/Home2005.htm. Accessed January 13, 2011.

9. Markovic D, Wang CC, Alarcon LP, Liu TT, Rabaey JM. Ultralowpower design in near-threshold region. Proc IEEE; 2010;98(2): 237-252.

10. Deng J. Device modeling and circuit performance evaluation for nanoscale devices: silicon technology beyond $45 \mathrm{~nm}$ node and carbon nanotube field effect transistors. Ph.D. dissertation. Stanford, CA: Stanford University Press; 2007.

11. Patil N, Lin A, Zhang J, et al. Scalable carbon nanotube computational and storage circuits immune to metallic and mis-positioned carbon nanotubes. IEEE Trans Nanotechnol. 2010;(99).

12. Patil N, Lin A, Myers ER, Wong HSP, Mitra S. Integrated wafer-scale growth and transfer of directional carbon nanotubes and misalignedcarbon-nanotube-immune logic structures. Proc Symp VLSI Technology Digest Tech Papers. 2008;205-206.

13. Patil N, Deng J, Lin A, Wong HSP, Mitra S. Design methods for misaligned and mis-positioned carbon-nanotube-immune circuits. IEEE Trans Comput Aided Des Integr Syst. 2008;27(10):1725-1736.

14. Patil N, Lin A, Myers E, et al. Wafer-scale growth and transfer of aligned single-walled carbon nanotubes. IEEE Trans Nanotechnol. 2009;8(4):498-504.

15. Kang SJ, Kocabas C, Ozel T, et al. High-performance electronics using dense, perfectly aligned arrays of single-walled carbon nanotubes. Nat Nanotechnol. 2007;2:230-236. 
16. Deng J, Patil N, Ryu K, et al. Carbon nanotube transistor circuits: circuit-level performance benchmarking and design options for living with imperfections. Proc International Solid State Circuits Conference (ISSCC). 2007:70-71.

17. Patil N, et al. Circuit-level performance benchmarking and scalability of carbon nanotube transistor circuits. IEEE Trans Nanotechnol. 2009;8(1):37-45.

18. Akinwande D, et al. Monolithic integration of CMOS VLSI and carbon nanotubes for hybrid nanotechnology application. IEEE Trans Nanotechnol. 2008;7(5):636-639.

19. Javey A, Guo J, Farmer DB, et al. Self-aligned ballistic molecular transistors and electrically parallel nanotube arrays. Nano Lett. 2004;4(7): 1319-1322.

20. Yao Z, Kane CL, Dekker C. High-field electrical transport in single-wall carbon nanotubes. Phys Rev Lett. 2000;84(13):2941-2944.

21. Mann D, Javey A, Kong J, Wang Q, Dai H. Ballistic transport in metallic nanotubes with reliable Pd ohmic contacts. Nano Lett. 2003;3(11): 1541-1544.

22. Cui Y, Zhong Z, Wang D, Wang WU, Lieber CM. High performance silicon nanowire field effect transistors. Nano Lett. 2003;3(2):149-152.

23. Stanford University CNFET Model Web site. 2008. Available from: http://nano.stanford.edu/model.php?id=23. Accessed January 13, 2011.

24. Mintmire JW, White CT. Universal density of states for carbon nanotubes. Phys Rev Lett. 1998;81(12):2506-2509.

25. Deng J, Wong HSP. A compact SPICE model for carbon-nanotube field-effect transistors including nonidealities and its application - part I: model of the intrinsic channel region. IEEE Trans Electron Devices. 2007;54(12):3186-3194
26. Deng J, Wong HSP. A compact SPICE model for carbon-nanotube field-effect transistors including nonidealities and its application part II: full device model and circuit performance benchmarking. IEEE Trans Electron Devices. 2007;54(12):3195-3205.

27. Lin S, Kim YB, Lombardi F. CNFET-based SRAM cell by dual-chirality selection. IEEE Trans Nanotechnol. 2010;9(1):30-37.

28. Aly R, Faisal M, Bayoumi A. Novel 7T SRAM cell for low power cache design. Proc IEEE International SOC Conf. IEEE; 2005 Sep: 171-174.

29. Berkeley Predictive Technology Model (PBTM). Available from: http:// www-device.eecs.berkeley.edu/ ptm/. Accessed January 13, 2011.

30. Amlani I, Lewis J, Lee K, et al. First demonstration of AC gain from a single-walled carbon nanotube common-source amplifier. Proc Int Electron Devices Meet. 2006:1-4.

31. Roy K, Mukhopadhyay S, Mahmoodi-Meimand H. Leakage current mechanisms and leakage reduction techniques in deep-submicrometer CMOS circuits. Proc IEEE; 2003;91(2):305-327.

32. Mukhopadhyay S, Raychoudhury A, Roy K. Accurate estimation of total leakage current in scaled CMOS logic circuits based on compact current modeling. Proc 40th Annual Design Automation Conference (DAC). Anahiem, CA: ACM; 2003 Jun 2-6:169-174.

33. BSIM4.2.1 MOSFET Model, BSIM Group, UC Berkeley. Avalilable from: http://www-device.eecs.berkeley.edu/bsim3/. Accessed January 13, 2011.

34. Calhoun BH, Chandrakasan AP. Static noise margin variation for sub-threshold SRAM in 65-nm CMOS. IEEE J Solid-State Circuits. 2006;41(7):1673-1679.
Nanotechnology, Science and Applications

\section{Publish your work in this journal}

Nanotechnology, Science and Applications is an international, peerreviewed, open access journal that focuses on the science of nanotechnology in a wide range of industrial and academic applications. It is characterized by the rapid reporting across all sectors, including engineering, optics, bio-medicine, cosmetics, textiles, resource sustainability

\section{Dovepress}

and science. Applied research into nano-materials, particles, nanostructures and fabrication, diagnostics and analytics, drug delivery and toxicology constitute the primary direction of the journal. The manuscript management system is completely online and includes a very quick and fair peer-review system, which is all easy to use. 